\title{
Rankin-Cohen Parantezi Yardımıyla Elde Edilen Hecke Eigenform Örnekleri
}

\author{
İlker İNAM ${ }^{1 *}$, Elif TERCAN ${ }^{2}$, Banu İREZ AYDIN ${ }^{3}$, Zeynep DEMİRKOL \\ ÖZKAYA ${ }^{4}$ (D)
}

Bilecik Şeyh Edebali Üniversitesi, Fen Edebiyat Fakültesi, Matematik Bölümü, 11200, Bilecik

Geliş / Received: 08/04/2020, Kabul / Accepted: 01/06/2020

\section{$\ddot{O} z$}

$\mathrm{Bu}$ çalışmada yarım tamsayı ağırlıklı Hecke eigenformların sistematik seçimi probleminin özel bir durumu çözüme kavuşturulmuştur. Öyle ki 17/2 ve 21/2 ağırlıklı Hecke eigenformlar, Rankin-Cohen parantezi yardımıyla belli ağırlıktaki Eisenstein serisi ve klasik teta serisi cinsinden ifade edilmiştir. İspatlar tanımlardan yola çıkarak temel lineer cebir metotları yardımıyla modüler formlar için verilen Sturm sınırı kullanılarak yapılmıştır. Eisenstein serilerinin basit bir bölen fonksiyonu yardımıyla ifade edilebilir olması ve klasik teta serisinde çok fazla boşluk olması ile türevlerinin de kolayca hesaplanabilmesi nedeniyle bu örnekler yardımıyla Hecke eigenformların çok sayıda Fourier katsayısı bilgisayarda Magma, Sage veya Pari/GP gibi uygun bir cebir yazılımı yardımıyla kolaylıkla ve çok hızlı bir şekilde hesaplanabilir.

Anahtar Kelimeler: Modüler formlar, Hecke eigenformlar, Rankin-Cohen parantezi

\section{Hecke Eigenform Examples Obtained by Rankin-Cohen Brackets}

\begin{abstract}
In this work, a special case of the systematic selection problem of half integral weight Hecke eigenforms is solved. Thus, 17/2 and 21/2 weight Hecke eigenforms are expressed in terms of Eisenstein series and classical theta series with the help of Rankin-Cohen brackets. The proofs were made using the Sturm bound for modular forms with the help of basic linear algebra methods based on definitions. Since the Eisenstein series can be expressed with the help of a simple divisor function and the classic theta series are lacunary and their derivatives can also be easily computed, many of these Hecke eigenforms' Fourier coefficients can be easily computed with a suitable algebra software such as Magma, Sage or Pari/GP on the computer.
\end{abstract}

Keywords: Modular forms, Hecke eigenforms, Rankin-Cohen bracket.

\section{Giriş}

Modüler formlar uzun yıllardır yaygın olarak çalışılmaktadır ve birçok uygulama alanı bulunduğundan oldukça ilgi çekmektedir. Matematikte sayılar teorisi, cebir, karmaşık analiz ve geometriyi bir araya getiren modüler formlar fizikte de sicim teorisindeki teorik hesaplamalarda karşımıza çıkar. Modüler formların aritmetiğine bakılacak olursa Swinnerton-Dyer kongrüansları (Swinnerton-Dyer 1973), Ramanujan'ın çalışmaları ve nihayetinde Sato-Tate sanısı (Barnet-Lamb vd., 2011) gibi Fourier 
katsayılarıyla ilgili problemler karşımıza çıkar. Fermat 1635 'te $x, y$ ve $z$ tamsayılar ve $n>3$ olmak üzere $x^{n}+y^{n}=z^{n}$ denkleminin aşikardan başka çözümünün olmayacağını iddia etmiş, diğer ortaya attığ 1 sayilar teorisi problemleri zamanla çözülmesine rağmen bu problem uzun y1llar çözülemediği için Fermat'in Son Teoremi adını almıştır. Öte yandan 1955-57'de Taniyama ve Shimura, Weil'in de katk1larıyla her bir eliptik eğriye bir modüler form karşılık geldiğini ortaya atmıştır. İşte tam olarak burada modüler formlar (tıpkı eliptik eğriler gibi) belki de popülerliğini borçlu olduğu durum ortaya çıkar: Fermat'ın Son Teoremi. 1984'te Gerhard Frey, $x^{n}+y^{n}=$ $z^{n}$ denklemiyle bağlantılı olarak ortaya çıkan "yapay" eliptik eğrilerin bir modüler formla eşleşmediğini iddia etmiştir. $\mathrm{O}$ halde Fermat'ın Son Teoremi yanlış ise TaniyamaShimura sanısı da yanlıştır. 1985'te Jean Pierre Serre tarafindan "Epsilon Sanısı" olarak formal bir hal alan problem 1987'de Ken Ribet'in Epsilon Sanısı'nı ispatlayarak Fermat'ın Son Teoremi'nin ispatına açılacak bir yol inşa etmiştir: Eğer Taniyama-Shimura Sanısı doğru ise Fermat'ın Son Teoremi de doğrudur. Nihayet Andrew Wiles 1994'te (yayım yılı 1995) her bir eliptik eğriyle bir modüler form eşleştiğini kanıtlayarak Fermat'in Son Teoremi'ne 359 yıl sonra son noktayı koymuştur. Modüler formların kümesi karmaşık sayılar cismi üzerinde sabit bir ağırlık için sonlu boyutlu bir vektör uzayı olduğundan her bir modüler form yani bu vektör uzayının elemanı kolaylıkla belirlenebilir. Tanım gereği önemli simetrileri olan modüler formların periyodik olduğu da dikkate alınırsa Fourier teorisi gereği sinüs ve kosinüs fonksiyonları cinsinden ifade edilebilirler. $\mathrm{Bu}$ ise bizi her bir f modüler formunun $f=\sum_{n=0}^{\infty} a^{n} q^{n}, \quad(q=$ $\left.e^{2 \pi i z}, \operatorname{Im} z>0\right)$ şeklinde bir Fourier serisi ile temsil edilebileceği sonucuna götürür. Bir vektör uzayında nasıl ki lineer dönüşümler ve özvektörler önemliyse modüler formların uzay1 üzerinde de Hecke operatörleri ve bunların özdeğerleriyle elde edilen Hecke eigenformları önemlidir. Birçok sonuç bu alt uzay üzerinde verilir. Yarım tamsayı ağırlıklı Hecke eigenformlar ise tamsayı ağırlıklı klasik modüler formlardan kimi zaman farkl1 özellikler sergiler. $\mathrm{Bu}$ tip Hecke eigenformların sistematik seçimi literatürde bir açık problem olup özellikle istatistiksel problemlerde çok sayıda Fourier katsayısı gerekli olduğundan bu problemin çözümü oldukça önemlidir. İki modüler formdan yeni bir modüler form elde edilmesine yarayan bir türev operatörü olan Rankin-Cohen parantezi yardımıyla yarım tamsayı ağırlıklı Hecke eigenformların sistematik seçimi, problemin çözümüne yapılabilecek yaklaşımlardan birisidir. Yarım tamsayı ağırlıklı Hecke eigenformların sistematik seçimi kadar çok sayıda Fourier katsayılarının hızlı bir şekilde hesaplanması da problemin başka bir boyutudur. (Kohnen ve Zagier 1981) Sayfa 177 'deki $\delta$ fonksiyonundan ilham alarak katsayılar1 kolayca hesaplanabilen birer modüler form olan Eisenstein serileri ile Fourier açılımında çok fazla boşluk bulunan teta serilerini kullanmak bu anlamda çok akılcıdır. Teta serisi 1/2 ağırlıklı bir modüler form olduğundan $k>2$ ( çift sayı) ağırlıklı Eisenstein serisi ile teta serisine birinci mertebeden Rankin-Cohen parantezi uygulanırsa tanım gereği $k+1 / 2+2$ ağırlıklı bir modüler form elde edilir, yani yarım tamsayı ağırlıklı bir modüler form elde edilmesinin yolu bu seçimlerdir. Ancak sonuç Hecke eigenform olmak zorunda değildir. Bunu yaparken dikkatli bir algoritma takip edilmelidir. Modüler form uzayının önemli ve ilgi çekici "aritmetik" bir özelliğe sahip olan alt uzayı olan Kohnen plus uzayının boyutunun 1 olduğu yerlerde Hecke eigenformlara ulaşmak daha kolaydır. 
$\mathrm{Bu}$ çalışmada 17/2 ve 21/2 ağırlıklı Hecke eigenformlar Rankin-Cohen parantezi yardımıyla formülize edilmiş olup kanıtlarda lineer cebir metotları ile Sturm sinırı kullanılmıştır. Farklı ağırlıklardaki Hecke eigenformlar için de bu metot kullanılabilir. Ancak farklı ağırlıklarda genelde Fourier katsayıların tamsayılar olmayabileceği de unutulmamalidir.

Giriş bölümünde yer alan tüm bilgiler modüler formlar için (Miyake 2006) ve (Koblitz 1984) gibi klasik kaynaklardan elde edilebilir. Fermat'1n Son Teoremi'nin ispatı için (Wiles 1995) incelenebilir.

\section{Materyal ve Metot}

$\mathrm{Bu}$ bölümde makalede kullanılacak kavramlar tanitilacaktır. Makale boyunca $\mathcal{H}$ ile karmaşık üst yarı düzlem, $\operatorname{PSL}(2, \mathbb{Z})$ ile katsayıları tamsayı ve $a d-b c=1$ olmak üzere $\frac{a z+b}{c z+d}$ biçimindeki lineer kesirli dönüşümlerin grubu ve $\Gamma$ ile modüler grup yani, $\quad \Gamma=S L(2, \mathbb{Z}):=\left\{\left(\begin{array}{ll}a & b \\ c & d\end{array}\right): a, b, c, d \in\right.$ $\mathbb{Z}$ ve $a d-b c=1\} \quad$ gösterilecektir. Aksi belirtilmedikçe $z$ değişkenleri $\operatorname{Im} z>0$ özelliğinde olacaktır.

Tanım 1. (Cohen-Strömberg, 2018) $k \in \mathbb{Z}$ olsun ve $F: \mathcal{H} \rightarrow \mathbb{C}$ fonksiyonu göz önüne alinsin.

1. Ĕger her $\gamma=\left(\begin{array}{ll}a & b \\ c & d\end{array}\right) \in \Gamma$ ve $z \in \mathcal{H}$ için

$$
F(\gamma(z))=(c z+d)^{k} F(z)
$$

oluyor ise $F$ 'ye $\Gamma$ için $k$ ağırlıklı zayıf modüler adı verilir.

2. Eğer $F, \mathcal{H}$ üzerinde analitik ve $\operatorname{Im}(z) \rightarrow \infty \quad$ yapıldığında $\quad|F(z)|$ sınırlı kalıyor ise $F^{\prime}$ ye $\Gamma$ için $k$ ağırlıklı modüler form denir. $\mathrm{Bu}$ özellikteki modüler formların kümesi $M_{k}(\Gamma)$ ile gösterilir.

3. Eğer $\operatorname{Im}(z) \rightarrow \infty$ yapıldığında $F(z)$ sıfıra yakınsıyor ise $F^{\prime}$ ye $\Gamma$ için $k$ ağırlıklı cusp form denir. $\mathrm{Bu}$ özellikteki cusp formların kümesi $S_{k}(\Gamma)$ ile gösterilir.

Tanım 2. (Cohen-Strömberg, 2018) $M_{k}(\Gamma)$ vektör uzayı olduğu için Hecke operatörleri

adı verilen lineer operatörler $M_{k}(\Gamma)$ üzerinde hareket eder. Daha açık bir ifadeyle verilen bir $k$ ağırlıklı $f(z)$ modüler formu için $m$. Hecke operatörünün etkisi aşağıdaki gibi verilir:

$T_{m} f(z)=m^{k-1} \sum_{\left(\begin{array}{ll}a & b \\ c & d\end{array}\right) \in \Gamma \backslash M_{m}}(c z+d)^{-k} f\left(\frac{a z+b}{c z+d}\right)$.

Burada $M_{m}$ determinant1 $m$ olan 2x2 tamsay 1 girdili matrislerin kümesini temsil eder.

Tanım 3. (Cohen-Strömberg, 2018) Her bir $m=1,2, \ldots$ için tüm $T_{m}$ Hecke operatörleri için özvektör olan modüler forma Hecke eigenform (özform) adı verilir. Açıktır ki, baş katsayıs1 1 olan eigenformlar tam olarak Hecke operatörlerinin özvektörü olur, yani $f$, Fourier açılımındaki sabit terimin katsayısı 1 olan bir Hecke eigenform için $a_{m}, m$. Fourier katsayısı olmak üzere $T_{m} f=a_{m} f$ dir.

Uyarı. $k$ bir tamsayı olmak üzere $k+\frac{1}{2}$ bir yarım tamsayı olarak adlandırılır. Tamsayı ağırlıklı modüler form tanımında $(c z+d)^{k}$ olarak gelen otomorfi çarpanı yarım tamsayı tanımında kompleks karekök fonksiyonu olarak geleceği için biraz daha dikkatli olmamiz gerekir. 
$z \in \mathcal{H}$ olmak üzere teta serisi $\theta(z):=1+$ $2 \sum_{n=1}^{\infty} q^{n^{2}}$ olarak tanımlanır. $\theta(z+2)=$ $\theta(z)$ ve $\theta\left(-\frac{1}{z}\right)=\sqrt{-i z} \theta(z)$ bağıntıları sağlanır. Karmaşık karekök fonksiyonunun bilinen dalı seçilerek her $\gamma \in \Gamma$ için $b, c \equiv$ $0(\bmod 2),\left(\frac{2 c}{d}\right)_{2}$ Legendre sembolü ve $\epsilon_{d}=$ 1 veya $i$ (sırasıyla $d \equiv 1$ veya 3 için) olmak üzere $\quad \theta(\gamma(z))=\left(\frac{2 c}{d}\right)_{2} \epsilon_{d}^{-1}(c z+d)^{\frac{1}{2}} \theta(z)$ elde edilir. $\tilde{\theta}(z)=\theta(2 z)$ seçilerek her $\gamma \in$ $\Gamma_{0}(4)$ için $j(\gamma, z):=\left(\frac{2 c}{d}\right)_{2} \epsilon_{d}^{-1}(c z+d)^{\frac{1}{2}}$ olmak üzere $\tilde{\theta}(\gamma(z))=j(\gamma, z) \tilde{\theta}(z)$ olur.

Tanım 4. (Koblitz, 1984) $k$ bir tamsayı olsun. Her bir cusp noktasinda analitik olan ve $\tilde{\theta}(\gamma(z))=j(\gamma, z)^{k+1 / 2} \tilde{\theta}(z)$ fonksiyonel eşitliğini sağlayan fonksiyonlara $\Gamma_{0}(4)$ için $k+1 / 2$ ağırlıklı modüler form yani yarım tamsayı ağırlıklı modüler form denir ve $M_{k+\frac{1}{2}} \Gamma_{0}(4)$ ile gösterilir.

Sonsuzda sıfır olan yarım tamsayı ağırlıklı modüler forma yarım tamsayı ağırlıklı cusp form denir. $\mathrm{Bu}$ özellikteki cusp formların uzayı $S_{k+\frac{1}{2}} \Gamma_{0}(4)$ ile gösterilir.

Burada $\Gamma_{0}(4)$ ile $\Gamma$ 'nın 4 seviyeli denklik alt grubu gösterilmektedir. Daha kesin olarak bu grup, $\quad \Gamma$ 'nın $\quad c \equiv 0(\bmod 4) \quad$ şeklindeki dönüşümlerinden oluşmaktadır. Yarım tamsayı ağırlıklı modüler formların yukarıdaki fonksiyonel eşitliği sağlayabilmesi için mutlaka $\Gamma_{0}(4)$ (veya daha genel olarak $N \in \mathbb{Z}$ olmak üzere $\left.\Gamma_{0}(4 N)\right)$ alt grubu üzerinde tanımlanmalıdır.

Yarım tamsayı ağırlıklı modüler formlar üzerinde de Hecke operatörleri tanımlanabilir. Aşağıdaki sonuç (Koblitz, 1984) sf. 207'de verilmiştir.

Teorem 1. (Koblitz, 1984) Yarım tamsayı ağırlıklı modüler formlar üzerinde $p^{2}$. Hecke operatörü $4 \mid N, p$ asal, $p \nmid N$ ve $k=2 \lambda+1$ pozitif tek tamsayı olmak üzere $T_{p^{2}}$ olarak gösterilir ve $T_{p^{2}} f(z)=\sum_{n=0}^{\infty} b_{n} q^{n}$ olarak tanimlanır. Burada $\quad b_{n}=a_{p^{2} n}+$ $\left(\frac{(-1)^{\lambda} n}{p}\right) p^{\lambda-1} a_{n}+p^{k-2} a_{n / p^{2}}$ şeklindedir.

Tanım 5. (Koblitz, 1984) $p$ asal olmak üzere tüm $T_{p^{2}}$ 'ler için özvektör olan modüler forma yarım tamsayı ağırlıklı modüler formlar için Hecke eigenform denir.

Tanım 6. (Cohen-Strömberg, 2018) $f$ ve $g$, $\mathcal{H}$ üst yarı düzleminde $C^{\infty}$ sinıfından sirasıyla $k$ ve $l$ ağırlıklı iki fonksiyon olsun; bu durumda $f$ ve $g$ fonksiyonunun $n$. Rankin Cohen parantezi $[f, g]_{n}=$ $\sum_{j=0}^{n}(-1)^{j}\left(\begin{array}{c}n+k-1 \\ j\end{array}\right)\left(\begin{array}{c}l+n-1 \\ n-j\end{array}\right) \mathfrak{D}_{\tau}^{n-j} \mathfrak{D}_{\tau}^{j}(g)$ eşitliği ile tanımlanır. Burada $\quad \mathfrak{D}_{\tau}(f):=\frac{1}{2 \pi i} \frac{\partial f}{\partial \tau}$ şeklindedir.

Ayrıca $f, k$ ağırlıklı ve $g, l$ ağırlıklı birer modüler form ise $(k, l$ tamsayı veya yarım tamsayı) $[f, g]_{1}=l g f^{\prime}-k f g^{\prime} \in M_{k+l+\frac{1}{2}}(\Gamma)$ olur.

Tanım 7. (Cohen-Strömberg, 2018) $k>2$ bir çift tamsayı olsun. $(m, n)$ aynı anda sıfır olmayan tamsayı çifti olmak üzere $k$ ağırlıklı Eisenstein serisi $z \in \mathcal{H}$ olmak üzere $G_{k}(z):=\sum_{m, n \in \mathbb{Z}} \frac{1}{(m z+n)^{k}}$ olarak tanımlanır.

Bazı $k$ çift tamsayıları için Eisenstein serileri şu şekildedir:

$$
G_{4}(z)=\frac{1}{240}+q+9 q^{2}+28 q^{3}+73 q^{4}+\cdots
$$

$G_{6}(z)=-\frac{1}{504}+q+33 q^{2}+244 q^{3}+1057 q^{4}+\cdots$

$G_{8}(z)=\frac{1}{480}+q+129 q^{2}+2188 q^{3}+\cdots$

Tanım 8. (Cohen-Strömberg, 2018) $k>2$ bir çift tamsayı olsun. $\mathrm{Bu}$ durumda $k$ - 
ağırlıklı normalleştirilmiş Eisenstein serisi $E_{k}(z)$ ile gösterilir ve $E_{k}(z)=1-$ $\frac{2 k}{B_{k}} \sum_{n=1}^{\infty} \sigma_{k-1}(n) q^{n} \quad$ olarak tanımlanır. Burada $B_{k}, k$. Bernoulli sayısını gösterir ve $\sigma$ bölen fonksiyonu $\sigma_{k-1}(n):=\sum_{d \mid n} d^{k-1}$ olarak tanımlanır.

Bazı normalleştirilmiş Eisenstein serileri aşağıdaki gibidir:

$$
\begin{gathered}
E_{4}(z)=1+240 q+2160 q^{2}+\cdots \\
E_{6}(z)=1-504 q-16632 q^{2}+\cdots \\
E_{8}(z)=1+480 q+61920 q^{2}+\cdots
\end{gathered}
$$

Aşağıda çalışmanın ana sonucunda da kullanılacak olan $1 / 2$ ağırlıklı teta serisine ait (Cohen-Strömberg, 2018) sf. 39-40'ta yer alan sonuç verilmiştir.

Teorem 2. (Cohen-Strömberg, 2018) $z \in$ $\mathcal{H}$ ve $q=e^{2 \pi i z}$ olmak üzere

$\theta(z)=1+2 \sum_{n=1}^{\infty} q^{n^{2}} \in M_{\frac{1}{2}} \Gamma_{0}(4)$ olur.

Modüler formların aritmetiğinin ilk adımı olan iki modüler formun eşitliği (CohenStrömberg, 2018) sf. 185 'te aşağıdaki teorem yardımıyla verilir.

\section{Teorem 3. (Cohen-Strömberg, 2018)}

$f=\sum_{n=0}^{\infty} a_{n} q^{n}, \quad g=\sum_{n=0}^{\infty} b_{n} q^{n} \in$ $M_{k}\left(\Gamma_{0}(N)\right)$ olsun. $d_{N}$ sayıs $\Gamma_{0}(N)$ 'in $P S L_{2}(\mathbb{Z})$ 'deki görüntüsünün indeksi olmak üzere $M:=\frac{k d_{N}}{12}$ sayısı tanımlansın. Eğer $0 \leq$ $i \leq M$ için $a_{i}=b_{i}$ ise bu takdirde $f=g$ olur. $M$ sayısına Sturm sınırı adı verilir. (Kumar ve Purkait 2014)'te yarım tamsayı ağırlıklı modüler formlar için Sturm sınırına benzer bir sınır verilmiştir.
Tanım 9. (Koblitz, 1984) $f=\sum_{n=1}^{\infty} a_{n} q^{n} \in$ $S_{k+\frac{1}{2}}\left(\Gamma_{0}(4)\right) \quad$ olsun. $n \equiv 2,3(\bmod 4)$ özelliğindeki $a_{n}$ Fourier katsayıları sıfır olan cusp formlar $S_{k+\frac{1}{2}}\left(\Gamma_{0}(4)\right)$ uzayının alt uzayını oluşturur. $\mathrm{Bu}$ uzaya Kohnen plus uzayı adı verilir ve $S_{k+\frac{1}{2}}^{+}\left(\Gamma_{0}(4)\right)$ ile gösterilir.

\section{Temel Sonuçlar ve İspatlar}

\section{Ana Teorem 1.}

$$
f_{1}=-\frac{84}{2 \pi i}\left(3 G_{6}(4 z) \theta^{\prime}(z)-G_{6}^{\prime}(4 z) \theta(z)\right) \in S_{\frac{17}{2}}^{+}\left(\Gamma_{0}(4)\right)
$$

bir Hecke eigenformdur.

İspat. $\quad\left[G_{6}(4 z), \theta\right]_{1} \quad$ Rankin-Cohen parantezini hesaplayalım. Tanım 6 gereği $g_{1}:=6 G_{6}(4 z) \theta^{\prime}(z)-\frac{1}{2} G_{6}^{\prime}(4 z) \theta(z)=$ $-\frac{1}{42} q-\frac{44}{21} q^{4}+8 q^{5}-88 q^{8}+\cdots(1)$ olur.

$q$-lu terimin katsayısı 1 olacak şekilde normalleştirme yapılırsa $f_{1}=-42 g_{1}$ elde edilir. Öte yandan $\operatorname{dim}\left(S_{21 / 2}\left(\Gamma_{0}(4)\right)=3\right.$ olup

$$
\begin{gathered}
u_{1}:=q+88 q^{4}-336 q^{5}+3696 q^{8}-5535 q^{9}+O\left(q^{12}\right) \\
\begin{aligned}
u_{2}:=q^{2}+4 q^{4} & -56 q^{5}+126 q^{6}-224 q^{7} \\
& +488 q^{8}-576 q^{9}+O\left(q^{12}\right) \\
u_{3}:=q^{3}-6 q^{4} & +20 q^{5}-56 q^{6}+124 q^{7} \\
& -220 q^{8}+352 q^{9}+O\left(q^{12}\right)
\end{aligned}
\end{gathered}
$$

olmak üzere $\left\{u_{1}, u_{2}, u_{3}\right\} S_{17 / 2}\left(\Gamma_{0}(4)\right)^{\prime}$ nin bir tabanıdır. Bu uzay için Sturm sınırı 4 olup (1) eşitliğine dikkat edilirse $f_{1}$ 'in $n \equiv$ 2,3 $(\bmod 4) \quad$ özelliğindeki Fourier katsayılarının sifir olması nedeniyle $f_{1} \in S_{17 / 2}^{+}$olur. Son olarak $S_{\frac{17}{2}}^{+}\left(\Gamma_{0}(4)\right)$ 'te bir tek Hecke eigenform mevcut olup bu formun $u_{1}$ yani $f_{1}$ formu olduğu kolayca görülebilir, bu da ispatı bitirir.

\section{Ana Teorem 2.}




$$
f_{2}:=\frac{30}{2 \pi i}\left(8 G_{8}(4 z) \theta^{\prime}(z)-\frac{1}{2} G_{8}^{\prime}(4 z) \theta(z)\right) \epsilon S_{21 / 2}^{+}\left(\Gamma_{0}(4)\right)
$$

bir Hecke eigenformdur.

\section{İspat.}

$\left[G_{8}(4 z), \theta(z)\right]_{1}$ Rankin - Cohen parantezini uygulayalım. Tanım 6 gereği

$g_{2}:=\left[G_{8}(4 z), \theta(z)\right]_{1}=8 G_{8}(4 z) \theta^{\prime}(z)-$

$\frac{1}{2} G_{8}^{\prime}(4 z) \theta(z)=\frac{1}{30} q-\frac{56}{30} q^{4}+12 q^{5}-456 q^{8}+$ $\left(\frac{18}{60}+1032\right) q^{9}+\cdots(2)$

olur. q-lu terimin katsayısı 1 olacak şekilde normalleştirme yapılırsa $f_{2}=30 g_{2}$ elde edilir. Öte yandan $\operatorname{dim}\left(S_{21 / 2}\left(\Gamma_{0}(4)\right)=4\right.$ olup

$$
\begin{gathered}
u_{1}:=q+24 q^{5}+1344 q^{6}-4480 q^{7}-1920 q^{8} \\
+4089 q^{9}+O\left(q^{12}\right) \\
\begin{aligned}
& u_{2}:=q^{2}+112 q^{5}-426 q^{6}+672 q^{7}+2176 q^{8} \\
&+ 6144 q^{9}+O\left(q^{12}\right)
\end{aligned} \\
\begin{array}{r}
u_{3}:=q^{3}+8 q^{5}-56 q^{6}+148 q^{7}-448 q^{8} \\
+
\end{array} \\
u_{4}:=q^{4}-6 q^{5}+24 q^{9}+O\left(q^{12}\right) \\
\left.-480 q^{9}+O 0 q^{7}+210 q^{8}\right)
\end{gathered}
$$

olmak üzere $\left\{u_{1}, u_{2}, u_{3}, u_{4}\right\} S_{21 / 2}^{+}$nin bir tabanıdir.

Bu uzay için Sturm sınırı 5 olup (2) eşitliğine dikkat edilirse $f_{2}=u_{1}-56 u_{4}$ olup $f_{2} \in S_{21 / 2}^{+}\left(\Gamma_{0}(4)\right)$ olduğu açktır. Kohnen plus uzayı tanımı gereği $f_{2}$ 'nin $n \equiv$ $2,3(\bmod 4) \quad$ özelliğindeki Fourier katsayılarının sıfır olması nedeniyle $f_{2} \in S_{21 / 2}^{+}$olur.

Son olarak $S_{21 / 2}^{+}\left(\Gamma_{0}(4)\right)$ 'te bir tek Hecke eigenform mevcut olup bu formun $f_{2}=u_{1}$ $56 u_{4}$ formu olduğu kolayca görülebilir, bu da ispatı bitirir.

\section{Sonuç ve Tartışma.}

$\mathrm{Bu}$ çalışmada Rankin-Cohen parantezi yardımıyla $17 / 2$ ve $21 / 2$ ağırlıklı Kohnen plus uzayında bulunan Hecke eigenformlar Eisenstein serileri ve teta serisi cinsinden ifade edilmiştir. Dikkat edilirse bu Hecke eigenformların en az $10^{8}$ Fourier katsayıs1 Magma (Bosma vd. 1997) Cebir Programı'nda kolaylıkla hesaplanabilir. $\mathrm{Bu}$ hesaplamada yalnızca çarpımlar yani Cauchy çarpımları zaman alır. Magma Cebir Programi'nda var olan Fast Fourier Transform (FFT) özelliği bu anlamda bu çarpımları kolaylaştırır. Eisenstein serileri ve özellikle teta serisinin oluşturulması herhangi bir hesaplama yükü getirmez. Sonuç olarak elde edilen bu basit formüller yardımıyla bu iki ağırlık için yeterince Fourier katsayısı kolayca hesaplanabilir.

Burada şunu da not düşmekte fayda var, Pari/GP (Pari/GP 2019) Cebir Programı'nın 2.11 versiyonunda bulunan modüler formlar paketi (mf) modüler formlarla hesaplama yapmak isteyen araştırmacılara önemli avantajlar sağlamıştır. Örneğin ispatlarda kullanılan Sturm sınırı pratik şekilde Pari/GP'de hesaplanabilirken Magma'da bu sinır1 direkt olarak hesaplayabilecek bir komut bulunmamaktadır. Ancak Pari/GP'de FFT özelliği bulunmadığı için örneğin Cauchy çarpımında büyük hesaplamalarda hafıza problemi çıkacaktır. 
Teşekkür: $\mathrm{Bu}$ çalışmanın birinci, ikinci ve dördüncü yazarı 118F148 no'lu Türkiye Bilimsel ve Teknolojik Araştırma Kurumu (TÜBİTAK) 1001 projesi tarafindan desteklenmektedir ve belirli bir kısmı ikinci yazarın yüksek lisans tezinin bir bölümünü oluşturmaktadır. $\mathrm{Bu}$ çalışma Covid-19 pandemisi sürecinde tamamlanmış olup tüm sağlık çalışanlarına armağan edilmiştir. Makaleyi dikkatli şekilde değerlendirip gelişimine katkıda bulunan hakemlere teşekkür ederiz.

\section{Kaynaklar}

Barnet-Lamb, T., Geraghty, D., Harris, M., Taylor, R. 2011 "A Family of Calabi-Yau varities and Potential Automorphy II", Publications of the Research Institute for Mathematical Sciences, 47, 1, 29-98.

Bosma W., Cannon J., Playsout C. 1997. The Magma algebra system I, The User Language. J. Symbolic Comput., 24, no. 3-4, 235-265.

Cohen, H., Strömberg, F. 2017., Modular Forms: A Classical Approach. Graduate Studies in Mathematics, 179., American Mathematical Society.
Koblitz, N.1984, "Introduction to Elliptic Curves and Modular Forms", Springer, Graduate Texts in Mathematics, 97.

Kohnen W., Zagier D. 1981. "Values of $L$ series of the modular forms at the center of the critical strip", Invent. Math., 64, 175-198.

Kumar, N., Purkait, S., A note on the Fourier coefficients of half-integral weight modular forms. (English summary) Arch. Math. (Basel) 102 (2014), no. 4, 369-378.

Miyake, T. 2006. "Modular Forms", Springer, $\mathrm{x}+335 \mathrm{pp}$.

Pari/GP Computer Algebra System 2019. https://pari.math.u-bordeaux.fr (Erişim Tarihi:05.04.2020)

Swinnerton-Dyer, H.P.F, 1973, "On 1-adic representations and congruences for coefficients of modular forms. In: Modular Functions of One Variable III", Springer Lecture Notes 350.

Wiles, A. 1995. "Modular elliptic curves and Fermat's last theorem", Ann. of Math., 141(2):443-551 